\title{
Editorial: The Mammary Stroma in Normal Development and Function
}

\author{
Pepper Schedin • Russell C. Hovey
}

Received: 12 August 2010 /Accepted: 23 August 2010 /Published online: 9 September 2010

(C) The Author(s) 2010. This article is published with open access at Springerlink.com

\begin{abstract}
The mammary gland can no longer be simply viewed as an organ composed of epithelial cells within a passive stromal microenvironment. Many lines of evidence have evolved to reinforce the notion that mammary epithelial cell growth, differentiation, lactation and progression to cancer involves bidirectional interactions between the epithelial population and its surrounding stroma. Within this stroma are numerous systems that are all capable of modulating epithelial function. In this context, the mammary stroma is not simply a depot of adipose tissue in which mammary epithelial cells undertake a unique growth and differentiation process, although adipocytes can impart numerous modulatory signals to epithelial cells, and vice versa. Rather, the stromal environment constitutes and supports a critical vasculature that supplies nutrients and endocrine cues, a lymphatic system that not only removes metabolites but also provides an intimate interface with the immune system, and an extracellular matrix scaffold in which epithelial cells grow, differen-
\end{abstract}

P. Schedin $(\bowtie)$

Department of Medicine, Division of Medical Oncology,

University of Colorado Denver,

MS8117, RC-1S, 8401K, 12801 E 17th Ave,

Aurora, CO 80045, USA

e-mail: Pepper.Schedin@ucdenver.edu

P. Schedin

University of Colorado Cancer Center,

Bldg 500, Suite 6004C, 13001 E 17th Place,

Aurora, CO 80045, USA

R. C. Hovey

Department of Animal Science,

The University of California, Davis,

2145 Meyer Hall, One Shields Avenue,

Davis, CA 95616, USA tiate and regress. Ultimately all of these components play a critical role in directing the epithelial phenotype during normal mammary gland growth and function. An increasing appreciation for these different systems demands a view of mammary epithelial cells in a much different light, and further necessitates the development of model systems that incorporate and integrate increasing complexity.

Keywords Mammary fat pad - Vasculature - Stroma . Lymphangiogenesis $\cdot$ Immune $\cdot$ Epithelial

Just over a decade ago an editorial article, "The mammary fat pad", appeared in this Journal wherein the state of knowledge regarding the adipocyte-rich stroma in which the mammary epithelium develops and functions was discussed [1]. Certainly prior to that 1998 editorial many important findings had been made regarding functions for the stromal environment within the mammary gland. Such roles were first recognized in the 1930's when Hass ([2], cited by [3]) mentioned developmental changes among adipocytes within the lactating mammary gland. Thereafter, the classic transplantation studies of Sakakura [4] Hoshino [5] and Kratochwil [6] showed critical requirements for the mesenchymal components during mammary gland development in mice. Similarly, the works of Bissell and others [7, 8] made it resoundingly clear that the extracellular matrix was not only important for genetic, but also morphogenic, responses by epithelial cells. The 1980's and 1990's then represented a period when the concepts of stromal-derived paracrine growth factors and roles for epithelial-stromalinteractions in mediating ovarian hormone action blossomed $[1,9,10]$. Since that 1998 issue there have been considerable 
advances as to how the mammary stroma is viewed. In that editorial, passing reference to stromal components such as the vasculature, the lymphatics and the associated immune compartment was made, primarily because they were known to be present, but were not widely-studied. The concept of stem cells was beginning to gain traction, as were technical advances such as microarrays and proteomics.

Fast-forward 11 years to this present issue. One might summarize the most recent advances within the field as representing a move towards understanding how epithelial cells within the mammary glands function in the context of a complex organ. Now, more than ever, the mammary gland is not viewed as simply an elegant collection of epithelial cells that can grow, differentiate, synthesize and secrete milk, involute, or become cancerous. Instead, the stromal environment contributes a vascular bed, immune surveillance, a lymphatic drainage, a unique adipose-rich fat pad, and an extracellular matrix-rich microenvironment, in which all components act in concert. It is the critical roles for these components in mammary gland development and function that inspired this issue. The notion that these different components are interrelated is illustrated by a conceptual "buckyball model" in Fig. 1, whereby all constituents affect all other components. The interdependence of all these components within the mammary glands is consistent with the fact that the 60-carbon "buckyball" molecule also has

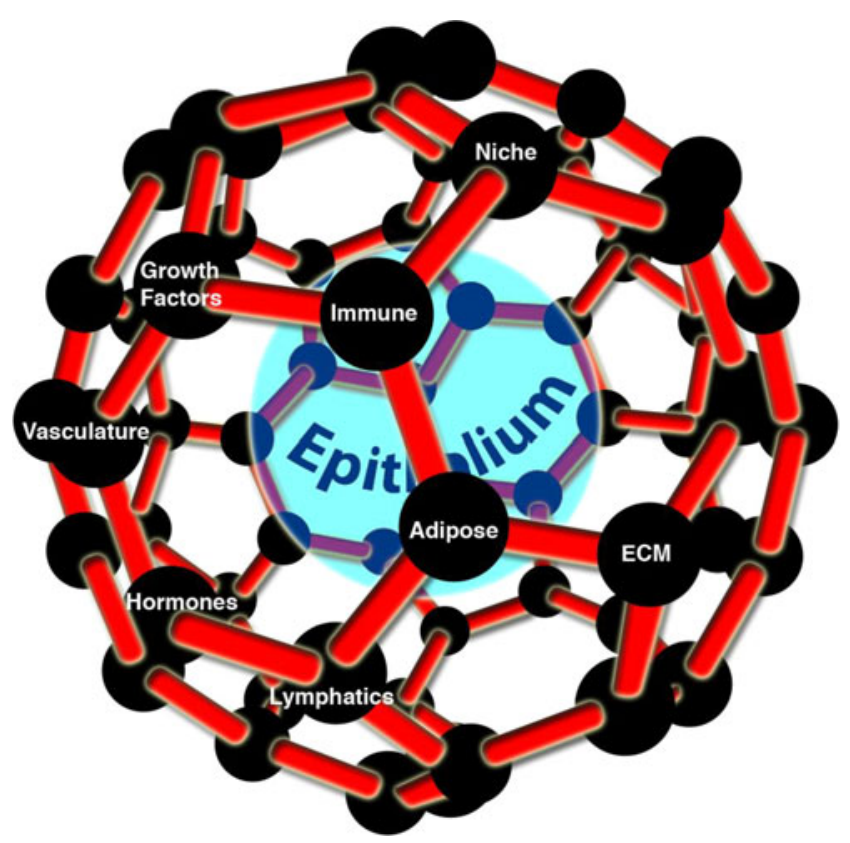

Figure 1 Modeling of the interrelated stromal components within the normal mammary glands as a hypothetical "buckyball" model. The 60-carbon buckyball has notable molecular stability, not unlike inherent tissue homeostasis in the normal mammary gland. Likewise, as depicted, the mammary gland also has several interrelated compartments that influence each other's behavior, centered about the epithelial population that grows, synthesizes and secretes milk, and becomes cancerous. No guarantees are provided regarding the molecular integrity of this buckyball. remarkable inherent stability. Figure 1 currently incorporates 8 stromal constituents that affect the adjacent mammary epithelium - hence, perhaps, there are only 52 more stromal influences to identify! Given this setting, an objective of this issue is to focus specifically on the normal mammary gland. Readers are also encouraged to also look forward to the December 2010 issue of this Journal where the roles for these same stromal components will be discussed in the context of breast cancer development and progression.

The mammary fat pad is an adipose-rich environment that, in many respects, forms a structural environment in which the epithelium grows and functions. It is this fat pad that also physically supports the intervening immune, lymphatic and vascular systems, in addition to the connective tissue extracellular matrix. In this issue, Hovey and Aimo [11] focus on the adipocytes and the many diverse potential roles they fulfill in the mammary gland during development, morphogenesis and function. Not only are there several mechanisms by which adipocytes might influence epithelial cells, but new data also indicate their stage-specific regulation. However, as highlighted by these authors, not all mammary fat pads are created equal across species, highlighting the need for further investigation into speciesspecificity in their role(s).

The vascular and lymphatic systems within the mammary gland are remarkably intricate. At the same time, both are critical for epithelial function. The importance of these components is perhaps most pronounced during lactation when there is concurrent delivery of nutrients (including large volumes of water) and removal of extracellular fluid and "waste" metabolites. An equally-important point to be considered is that both the vasculature and lymphatics develop in close association with the adjacent epithelial structures. Andres and Djonov [12] review development of the vasculature in the mammary glands and illustrate its fascinating complexity. From their discussion it becomes clear that numerous factors likely regulate vasculogenesis during the distinct phases of mammary gland development and function. Likewise, the lymphatics within the mammary gland are equally-crucial. While they play key roles during lactation and for clinical prognosis of breast cancer, surprisingly little is known about the full process of lymphangiogenesis, particularly as it applies to the normal mammary gland. None-the-less, Lund and Swartz provide a detailed overview of lymphatic function that provides compelling points of focus for future study of the mammary glands [13]. In turn, the lymphatics serve as a conduit for the immune system to interact with the mammary epithelium. In this light, Reed and Schwertfeger address the increasinglycomplex roles for immune cells in mediating normal mammary land development and function [14]. All cells of the mammary gland also interface with extracellular matrix (ECM) proteins. The complexities of the extracellular matrix 
microenvironment, its developmental regulation, and its potential interface with adipocytes, the vasculature, immune cells as well as epithelium are outlined by Maller et al. [15]. All of these relationships ultimately create a microenvironment in which epithelial cells develop and function. As has also become clear in this past decade, the epithelium includes a subset of stem cells that confers many unique properties to the mammary glands. As discussed by McCave et al. these stem cells also closely interact with the many faces of their microenvironment which, in turn, can alter their fate and the ultimate mammary gland phenotype [16].

The fascinating and beautiful-yet-complex stromal environment in the normal mammary gland does present challenges. Namely, the scientific art of reductionism and mechanistic study becomes exponentially-more difficult as more and more systems interact. While cell culture models have afforded numerous breakthroughs, new approaches in this realm also present opportunities to recapitulate these many components in vitro. One such technique that presents many new opportunities for studying mammary gland biology as well as for breast reconstruction is the silk-based model reviewed by Wang et al. [17]. The ability to more closelyrecapitulate the stromal environment is also addressed by O'Brien et al. in their article that describes methods for purifying mammary-specific extracellular matrix for use in such studies [18]. Modeling approaches such as these should further help to realize the components and relationships at play in the buckyball model of the mammary gland.

Advances in our understanding of the stromal environment raises further questions for the future about how this dynamic organ functions. For example, looking ahead, will it be possible to fully recapitulate all of these interacting systems in order to study the interrelationships between the epithelium and the other stromal constituents? Can the greatest insight be obtained by developing increasingly complex in vitro systems or by even higher resolution in vivo imaging? Regardless, technological advances such as these, and those of the future, will continue to pave the way for many discoveries involving all aspects of mammary gland growth and function.

Ultimately, as this issue seeks to highlight, the mammary gland is a complex organ that involves numerous tissue compartments. Now that many of these systems are known and appreciated, one important consideration remainsnamely that, as with any biological system, there is ongoing flux between these compartments and the greater organism. The mammary glands are also part of the entire organism and depend on close bi-directional communication in this setting. Needless to say, maternal influence, environmental exposures, and modifiable lifestyle factors (such as diet and exercise) add extra dimensions that present new challenges for studying this system. At the same time, these avenues of research represent exciting opportunities to delve further into the many aspects of mammary gland growth and function.
Open Access This article is distributed under the terms of the Creative Commons Attribution Noncommercial License which permits any noncommercial use, distribution, and reproduction in any medium, provided the original author(s) and source are credited.

\section{References}

1. Tonelli QJ, Sorof S. Epidermal growth factor requirement for development of cultured mammary gland. Nature. 1980;285 (5762):250-2.

2. Hass E. Die Beziehungen zwischen Drusengewebe und zugehorigem Fettlager bei der Milchdruse der Maus. Z. mikroskop. Anat Forsch. 1933;34:201-37.

3. Elias JJ, Pitelka DR, Armstrong RC. Changes in fat cell morphology during lactation in the mouse. Anat Rec. 1973;177(4):533-47.

4. Sakakura T, Nishizuka Y, Dawe CJ. Mesenchyme-dependent morphogenesis and epithelium-specific cytodifferentiation in mouse mammary gland. Science. 1976;194(4272):1439-41.

5. Hoshino K. Morphogenesis and growth potentiality of mammary glands in mice. I. Transplantability and growth potentiality of mammary tissue of virgin mice. J Natl Cancer Inst. 1962;29:835-51.

6. Kratochwil K. Organ specificity in mesenchymal induction demonstrated in the embryonic development of the mammary gland of the mouse. Dev Biol. 1969;20(1):46-71.

7. Lee EY, Parry G, Bissell MJ. Modulation of secreted proteins of mouse mammary epithelial cells by the collagenous substrata. J Cell Biol. 1984;98(1):146-55.

8. Emerman JT, Pitelka DR. Maintenance and induction of morphological differentiation in dissociated mammary epithelium on floating collagen membranes. In Vitro. 1977;13(5):316-28.

9. Ruan W, Newman CB, Kleinberg DL. Intact and amino-terminally shortened forms of insulin-like growth factor I induce mammary gland differentiation and development. Proc Natl Acad Sci USA. 1992;89(22):10872-6.

10. Sasaki M, Nishio M, Sasaki T, et al. Identification of mouse mammary fibroblast-derived mammary growth factor as hepatocyte growth factor. Biochem Biophys Res Commun. 1994;199(2):772-9.

11. Hovey RC, Aimo L. Diverse and active roles for adipocytes during mammary gland growth and function. J Mammary Gland Biol Neoplasia 2010. doi:10.1007/s10911-010-9187-8

12. Andres AC, Djonov V. The mammary gland vasculature revisited. J Mammary Gland Biol Neoplasia 2010. doi:10.1007/s10911-010-9186-9

13. Lund AW, Swartz MA. Role of lymphatic vessels in immunity: Passive conduits or active participants? Journal of Mammary Gland Biology and Neoplasia 2010. doi:10.1007/s10911-010-9193-x

14. Reed JR, Schwertfeger KL. Immune cell location and function during post-natal mammary gland development. J Mammary Gland Biol Neoplasia 2010. doi:10.1007/s10911-010-9188-7

15. Maller O, Martinson H, Schedin P. Extracellular matrix composition reveals complex and dynamic stromal-epithelial interactions in the mammary gland. Journal of Mammary Gland Biology and Neoplasia 2010. doi:10.1007/s10911-010-9189-6

16. McCave E, Cass CAP, Burg JL, Booth BW. The normal microenvironment directs mammary gland development. Journal of Mammary Gland Biology and Neoplasia 2010. doi:10.1007/ s10911-010-9190-0

17. Wang X, Reagan M, Kaplan DL. Synthetic adipose tissue models for studying mammary gland development and breast tissue engineering. Journal of Mammary Gland Biology and Neoplasia 2010. doi:10.1007/s10911-010-9192-y

18. O'Brien J, Fornetti J, Schedin P. Isolation of mammary-specific extracellular matrix to assess acute cell-ECM interactions in 3D culture. Journal of Mammary Gland Biology and Neoplasia 2010. doi:10.1007/s10911-010-9185-x 